\title{
An Apriori Algorithm-Based Association Rule Analysis to Identify Herb Combinations for Treating Uremic Pruritus Using Chinese Herbal Bath Therapy
}

\author{
Ping-Hsun Lu, ${ }^{1,2}$ Jui-Lin Keng, ${ }^{3}$ Ko-Li Kuo, ${ }^{4,5}$ Yu-Fang Wang, ${ }^{1}$ Yu-Chih Tai, ${ }^{1,2}$ \\ and Chan-Yen Kuo ${ }^{6}{ }^{6}$ \\ ${ }^{1}$ Department of Chinese Medicine, Taipei Tzu Chi Hospital, Buddhist Tzu Chi Medical Foundation, New Taipei, Taiwan \\ ${ }^{2}$ School of Post-Baccalaureate Chinese Medicine, Tzu Chi University, Hualien, Taiwan \\ ${ }^{3}$ Department of Applied Mathematics, University of Washington, Seattle, WA, USA \\ ${ }^{4}$ Division of Nephrology, Department of Internal Medicine, Taipei Tzu Chi Hospital, Buddhist Tzu Chi Medical Foundation, \\ New Taipei, Taiwan \\ ${ }^{5}$ School of Medicine, Tzu Chi University, Hualien, Taiwan \\ ${ }^{6}$ Department of Research, Taipei Tzu Chi Hospital, Buddhist Tzu Chi Medical Foundation, New Taipei City, Taiwan
}

Correspondence should be addressed to Yu-Chih Tai; tyc5326@gmail.com and Chan-Yen Kuo; cykuo863135@gmail.com

Received 29 September 2020; Revised 23 October 2020; Accepted 24 October 2020; Published 23 November 2020

Academic Editor: Priscila Souza

Copyright (C) 2020 Ping-Hsun Lu et al. This is an open access article distributed under the Creative Commons Attribution License, which permits unrestricted use, distribution, and reproduction in any medium, provided the original work is properly cited.

Uremic pruritus (UP) is prevalent among patients with end-stage renal disease (ESRD), which causes severe itching and affects their quality of life. Additionally, patients experience fatigue and depression, and an increased risk of mortality has also been reported. A meta-analysis of 17 randomized controlled trials (RCTs) has indicated that Chinese herbal bath therapy (CHBT) had adjuvant benefits in improving UP in ESRD patients, and previous studies have reported that herb combinations were more useful than treatment with a single herb. Association rule analysis has been used to evaluate potential correlations between herb combinations, and Apriori algorithms are one of the most powerful machine-learning algorithms available for identifying associations within databases. Therefore, we used the Apriori algorithm to analyze association rules of potential core herb combinations for use in CHBT for UP treatment using data from a meta-analysis of 17 RCTs that used CHBT for UP treatment. Data on 43 CHBT herbs were extracted from 17 RCTs included for analysis and we found 19 association rules. The results indicated that the following herb combinations \{Chuanxiong, Baijili $\} \geq\{$ Dahuang $\}$ and $\{$ Dahuang, Baijili $\} \geq\{$ Chuanxiong $\}$ were most strongly associated, implying that these herb combinations represent potential CHBT treatments for UP.

\section{Introduction}

Uremic pruritus (UP) is a common but annoying symptom among patients with advanced chronic kidney disease (CKD) or on dialysis; it affects $24 \%$ of nondialysis CKD patients and $55 \%$ of dialysis patients $[1,2]$. UP may be generalized or localized and typically worsens at night, thereby affecting sleep quality. The persistence of these symptoms leads to patients becoming fatigued, anxious, and depressed. Furthermore, the risk of mortality increases due to chronic fatigue syndrome $[3,4]$. The pathogenesis of UP is not fully understood but systemic inflammation and opioid system imbalance are thought to be important contributors $[3,5,6]$. Additionally, effective treatment strategies for UP are lacking as its pathophysiology is not completely understood. Current therapies like antihistamines, gabapentin, nalfurafine, and phototherapy not only have a limited therapeutic effect but are also associated with adverse effects, such as dizziness, drowsiness, and somnolence [7, 8]. Therefore, alternative treatments for UP are required.

Complementary and alternative medicine is an attractive treatment option for UP because it is cheap and can have 
fewer side effects [9]. Chinese herbal bath therapy (CHBT) is an ancient treatment method that has been broadly used in China for thousands of years to ameliorate symptoms of certain diseases, including UP in uremic patients $[7,8]$. The medicinal ingredients used in CHBT can improve blood circulation and have been shown to have analgesic and antiinflammatory effects $[10,11]$. A recent meta-analysis has revealed $\mathrm{CHBT}$ to be effective as an adjunct therapy for improving UP among ESRD patients and the possible underlying mechanisms include improved blood circulation to promote metabolism, elimination of uremia-associated toxins $[7,12]$, and anti-inflammatory effects $[10,13]$. It is widely recognized that both selection and combination of herbs used in CHBT are crucial for its success, and the principles used for selecting and combining such herbs follow ancient theories provided in the Rhymed Discourse for Topical Remedies and prescriptions of Chinese Materia Medica $[14,15]$. Nevertheless, there is no clear consensus on the standard of CHBT herbs, or their combinations, for use in UP treatment.

Data mining methods have recently gained broad application in Chinese medicine, and many studies have used data mining to provide benchmarks for selecting and combining herbs for treating CKD [16], bone marrow suppression [17], and breast cancer recurrence and metastasis [18]. Even though the clinical practice of prescribing herbal medications is based on combinations of herbs, criteria for determining requisite combinations are lacking. Thus, association rule analysis could be used as an essential and practical approach for exploring the fundamental rules that would yield effective herb combinations. In recent years, machine-learning algorithms have been applied in many fields, including Chinese medicine. Apriori, an association rule learning algorithm, can determine the frequency of sets of items in databases [19] and also the importance of association rules based on certain metrics, such as support, confidence, and lift. Apriori offers thorough association analysis and can provide insights into trends within a database [19].

Hence, we aimed to explore potential core herb combinations for CHBT that can be used in the treatment of UP using an Apriori algorithm-based association rule analysis on datasets derived from a meta-analysis of 17 randomized controlled trials (RCTs) [8].

\section{Materials and Methods}

2.1. Data Sources and Selection Criteria. This analysis was based on the previously reported meta-analysis of 17 RCTs on CHBT treatment for UP [8]. We extracted data on herbs used for CHBT from these 17 RCTs collected from 2004 to 2017.

This meta-analysis included 970 UP patients with advanced CKD or those who were on dialysis; patients were graded according to the diagnostic criteria of the Chinese diagnostic standard or National Kidney Foundation (NKF) [8]. All the included studies were required to have precise outcome data on UP assessment parameters and to have conducted the herbal bath with only the patient's head remaining out of the water. The exclusion criteria were the use of other traditional Chinese medicine treatments by the patients or incomplete outcome data. The CHBT prescription uses an average of 11 Chinese herbs; the average duration of CHBT treatment was 4.7 weeks. Compared with the control group, CHBT has significantly improved visual analog scale (VAS) and symptom scores.

2.2. Risk of Bias Assessment. The methodological quality of the studies included for the meta-analysis was assessed using the Cochrane risk of bias (RoB) 2.0 tool [20]. This tool has five domains for assessing the risk of bias and the responses to questions in each domain are combined to provide an estimate of the overall quality of the RCT. This quality assessment procedure has been described in detail previously [8].

2.3. Data Analysis. We investigated the frequency of use of Chinese medicinal herbs. The software RStudio (version 1.2.5033, Integrated Development for R. RStudio, PBC, Boston, MA) was used to conduct the Apriori association rule learning analysis and to plot charts [21]. Data was processed into 17 columns with each column representing one CHBT formula, and data were fitted using the R package "arules." Additionally, charts were produced and visualized by fitting data into the R package, "arulesViz."

Association rule learning algorithms are the most popular method of identifying and analyzing trends or relations within transaction data [22]. Many studies have utilized association rule learning algorithms to detect hidden relations in medical fields [16-18]. Generally, an Apriori algorithm connects an antecedent set of items to a consequent set of items based on the notion that these two sets of items only occur together in the database, rather than due to a causal effect.

Support, confidence, expected confidence, and lift are four standard metrics used to measure associations between items in the Apriori algorithm. Support measures the proportion of CHBT formulas in which a certain herb appears while confidence measures the proportion of CHBT formulas with herb A, in which herb B also appears. Next, expected confidence measures the percentage of occurrences containing the consequent in a relation and represents the probability of the consequent if it is independent of the antecedent. Lastly, lift is the results obtained when confidence is divided by expected confidence and represents the likelihood of an increase in the consequent given a particular antecedent. In other words, lift quantifies the probability of herb $\mathrm{B}$ appearing when herb $\mathrm{A}$ is present in a CHBT formula while controlling for expected confidence.

We analyzed the 19 best association rules with the minimum values for support and confidence being $5 \%$ and $90 \%$, respectively. The reason for choosing these criteria was that the highest value for support was only 0.13636364 . Additionally, the values of confidence for overall association rules were above 0.5 . Based on the minimum requirements of the metrics, we filtered out less significant association rules and found that 19 association rules remained in the 
dataset. Lastly, as the confidence values for all 19 association rules in this study were unity, the association rules were sorted in descending order based on values obtained for the metric "support".

\section{Results}

3.1. Study Characteristics and Risk of Bias Assessment. The methodologic quality of the retrieved studies is summarized in Table 1 and a detailed RoB assessment is provided in Supplementary Figure 1 [8]. The overall quality of the 17 RCTs was variable, with only two of them determined to be "moderate." Possible reasons include the fact that only one article described a specific grouping method, none of the retrieved studies described allocation concealment, and only one trial used patient blinding.

3.2. Distribution of the Herb. We identified 43 herbs from the 17 RCTs included in the aforementioned meta-analysis. The frequency distribution of herbs is shown in Figure 1 and the Latin scientific names of the species are shown in Table 2. The 10 most-frequently selected herbs for use in CHBT for treating UP were Difuzi, Baixianpi, Kushen, Chantui, Danggui, Xixin, Chuanxiong, Jingjie, Tufulin, and Dahuang.

\subsection{Apriori Algorithm-Based Association Rule Analysis for} Item Sets of Herbs Combinations. We analyzed 19 association rules based on data from 17 Chinese-medical-herb formulas. The association rules were converted to a scatter plot with support values on the $x$-axis and confidence values on the $y$ axis. The color of each association rule was determined by its lift value (Figure 2). We found that all association rules had a relatively high lift, indicating that the likelihood of antecedent herb and consequent herb being in an association rule together was multiple times that of the consequent herb when used alone. The confidence values for all 19 association rules was one, implying that each pair of the antecedent and the consequent herb appeared together whenever the antecedent herb was present in a formula in all 19 association rules. In contrast, only two values were obtained for support (0.13636364, 0.09090909); therefore, as seen in Figure 2, there was overlap among the 16 association rules on the lefthand side and among the three association rules on the right-hand side. These observations on support values revealed that the antecedent herb in each association rule did not frequently show in the 19 formulas. Thus, we deduced that each formula used for treating UP was implemented independently. All 19 association rules are listed in Table 3.

A grouping matrix diagram, which displays the general distribution of clustered association rules, showed similar cluster-grouping of association rules (Figure 3 ). The horizontal ordinate represents 9 clusters, and the vertical ordinate stands for items produced by the 9 clusters (rules). The depth of color within a circle represents the degree of lift such that the darker the color, the higher the degree of lift. Circle size represents the degree of support such that the larger the circle, the higher the degree of support. The herb combinations $\{$ Chuanxiong, Baijili $\} \geq\{$ Dahuang $\}$,
$\{$ Dahuang, Baijili $\} \geq\{$ Chuanxiong $\}$ were interactively selected and they revealed the association rules connecting from antecedent (LHS) to consequent (RHS) item sets. The results of these interactively selected association rules consistently corresponded with association rules \#4 $\{$ Chuanxiong $\} \geq\{$ Dahuang $\}$ and $\#$ 5 Dahuang $\} \geq\{$ Chuanxiong\}, as seen in Table 3 .

\section{Discussion}

Our results indicate that Chuanxiong, Baijili, and Dahuang, and Dahuang, Baijili, and Chuanxiong were the most-frequently used core herb combinations in CHBT for UP. Importantly, a meta-analysis of CHBT for UP revealed that the herb combination used in CHBT played a significant role in improving UP symptoms in patients with advanced CKD [8]. The usefulness of evidence-based strategies for selecting herbs for further treatment can be determined by their efficacy, and to the best of our knowledge, this is the first report on potential core herb combinations in CHBT for UP.

The current studies confirm that Chinese medicinal formulas containing herb combinations, when used orally or externally, are beneficial for patients with UP and that the possible underlying mechanisms include anti-inflammatory effects $[10,13]$, accelerating blood circulation to promote metabolism and eliminating uremia toxins [7, 12]. Chen et al. have reported a RCT that combined hemodialysis and an oral uremic clearance granule (UCG) containing 16 herbs, such as Chuanxiong, Dahuang, Kushen, Danshen, Heshouwu, and Sangbaipi, among others. They state that these herbs could not only significantly reduce itching when measured using a visual analog scale but also ameliorate renal function and reduce serum levels of phosphorus, calcium, intact parathyroid hormone (iPTH), and inflammatory biomarker, such as beta 2-microglobulin ( $\beta 2-\mathrm{MG})$ [9]. Additionally, Kao et al. have revealed that a UCG-enema group showed a better effective rate for itch relief compared to the control group [40]. Wang et al. have demonstrated that Xiao Feng San (XFS), a traditional Chinese formula composed of 13 herbs, namely, Kushen, Chantui, Danggui, Fangfeng, and Jingjie, was better able to reduce pruritus and parathyroid hormone levels compared to control [41]. Bai et al. conducted an RCT using life paste, a Chinese herbbased cream that contains Dahuang, Danggui, Zicao, and Shechuangzi, and showed a better antipruritic effect for the herb combination than the lotion without the additives [42]. The Latin scientific names of the species mentioned above are shown in Table 2. The combination of Astragalus henryi Oliv. and Angelica sinensis (Oliv.) Diels (A\&A) have been shown to improve renal blood flow in rats with acute ischemic renal injury [43] and to reduce capillary loss; the latter involved higher expression of the vascular endothelial growth factor (VEGF) [44].

Many studies have reported that herb pairs exhibit greater pharmacological efficacy than a single herb. Specifically, Ying et al. describe that the Alpinia oxyphylla-Schisandra chinensis herb pair (ASHP) has an anti-Alzheimer's disease effect via ameliorating abnormal changes in cognitive behavior, and that ASHP can improve the absorption of and slow down the 
TABLE 1: Summary of 17 RCTs included in the meta-analysis on CHBT treatment for UP.

\begin{tabular}{|c|c|c|c|c|}
\hline Study (year) & $\begin{array}{l}\text { Study } \\
\text { design }\end{array}$ & $\begin{array}{l}\text { Inclusion } \\
\text { criteria }\end{array}$ & Herbs & $\begin{array}{c}\text { Overal } \\
\text { bias }\end{array}$ \\
\hline Du, 2009 [23] & $\mathrm{RCT}$ & $\mathrm{HD}$ & $\begin{array}{c}\text { Tufulin, chuanxiong, baizhi, baixianpi, difuzi, kushen, jingjie, xixin, danggui, } \\
\text { chantui }\end{array}$ & High \\
\hline $\begin{array}{l}\text { Du et al., } 2004 \\
{[24]}\end{array}$ & RCT & $\mathrm{HD}$ & Tufulin, chuanxiong, baixianpi, difuzi, heshouwu, kushen, jingjie, xixin, danggui & High \\
\hline $\begin{array}{l}\text { Gao and Ye, } \\
2012[25]\end{array}$ & $\mathrm{RCT}$ & $\mathrm{HD}$ & $\begin{array}{l}\text { Tufulin, dahuang, baixianpi, difuzi, heshouwu, fangfeng, taoren, mahuang, } \\
\text { danshen, jixueteng }\end{array}$ & High \\
\hline $\begin{array}{l}\text { Guo et al., } 2009 \\
{[26]}\end{array}$ & RCT & HD & $\begin{array}{c}\text { Dahuang, chuanxiong, danshen, baixianpi, difuzi, honghua, kushen, xixin, } \\
\text { mahuang, danggui }\end{array}$ & High \\
\hline $\begin{array}{l}\text { Jia et al., } 2012 \\
\text { [27] }\end{array}$ & $\mathrm{RCT}$ & $\mathrm{HD}$ & $\begin{array}{l}\text { Dahuang, chuanxiong, baizhi, baixianpi, difuzi, zaojiaoci, kushen, xixin, } \\
\text { danggui, chantui, jixueteng }\end{array}$ & High \\
\hline $\begin{array}{l}\text { Jia, Li and Fei, } \\
2012[28]\end{array}$ & RCT & $\mathrm{HD}$ & $\begin{array}{l}\text { Dahuang, chuanxiong, baizhi, baixianpi, difuzi, zaojiaoci, kushen, xixin, } \\
\text { danggui, chantui, jixueteng }\end{array}$ & High \\
\hline Lan, $2017[29]$ & $\mathrm{RCT}$ & $\mathrm{HD}$ & $\begin{array}{c}\text { Dahuang, chuanxiong, danshen, baixianpi, difuzi, honghua, kushen, xixin, } \\
\text { mahuang, danggui }\end{array}$ & High \\
\hline Lin, 2014 [30] & $\mathrm{RCT}$ & $\mathrm{HD}$ & $\begin{array}{l}\text { Dahuang, chuanxiong, baizhi, baixianpi, difuzi, zaojiaoci, kushen, xixin, } \\
\text { danggui, chantui, jixueteng }\end{array}$ & High \\
\hline Shen, 2014 [31] & RCT & $\mathrm{HD}$ & $\begin{array}{l}\text { Tufulin, danshen, baixianpi, quanxie, difuzi, heshouwu, kushen, xixin, } \\
\text { shechuangzi, mahuang, danggui, wugong, chantui, biejia }\end{array}$ & High \\
\hline $\begin{array}{l}\text { Wang et al., } 2013 \\
{[32]}\end{array}$ & $\mathrm{RCT}$ & $\mathrm{HD}$ & $\begin{array}{c}\text { Dahuang, chuanxiong, baixianpi, difuzi, chishao, kushen, xixin, shechuangzi, } \\
\text { juhua, danggui, chantui }\end{array}$ & High \\
\hline Wen, 2007 [33] & RCT & $\mathrm{HD}$ & Tufulin, chuanxiong, baijili, difuzi, wushaoshe, jingjie, xixin, danggui, jixueteng & High \\
\hline Yao, 2015 [34] & RCT & $\mathrm{HD}$ & Tufulin, baixianpi, difuzi, kushen, jingjie, chantui & High \\
\hline $\begin{array}{l}\text { Yu et al., } 2017 \\
\text { [35] }\end{array}$ & RCT & $\mathrm{HD}$ & $\begin{array}{l}\text { Tufulin, chuanxiong, baixianpi, difuzi, kushen, jingjie, zisuye, danggui, jixuecao, } \\
\text { chantui }\end{array}$ & High \\
\hline $\begin{array}{l}\text { Zhang et al., } \\
2014[36]\end{array}$ & $\mathrm{RCT}$ & $\mathrm{HD}$ & Tufulin, difuzi, baixianpi, kushen, chantui, jingjie & High \\
\hline $\begin{array}{l}\text { Zhang et al., } \\
2012[37]\end{array}$ & $\mathrm{RCT}$ & $\mathrm{HD}$ & $\begin{array}{l}\text { Baizhi, baijili, fangfeng, songjie, qianghuo, guizhi, jingjie, xixin, shechuangzi, } \\
\text { mahuang, cangzhu, tanxiang, chantui }\end{array}$ & High \\
\hline Zhao, 2011 [38] & RCT & $\mathrm{HD}$ & $\begin{array}{l}\text { Wujiapi, baizhi, baijili, shenjincao, fangfeng, peilan, songjie, qianghuo, } \\
\text { weilingxian, guizhi, taozhi, haitongpi, jingjie, xixin, shechuangzi, mahuang, } \\
\text { zisuye, huaizhi, cangzhu, tanxiang, chantui, huoxiang }\end{array}$ & Some \\
\hline Zheng, 2016 [39] & RCT & HD & $\begin{array}{l}\text { Chuanxiong, shengdihuang, difuzi, chishao, fangfeng, kushen, mahuang, } \\
\text { danggui, chantui }\end{array}$ & High \\
\hline
\end{tabular}

$\mathrm{RCT}=$ randomized controlled trial; $\mathrm{CHBT}=$ Chinese herbal bath therapy; $\mathrm{UP}=$ uremic pruritus; $\mathrm{HD}=$ hemodialysis.

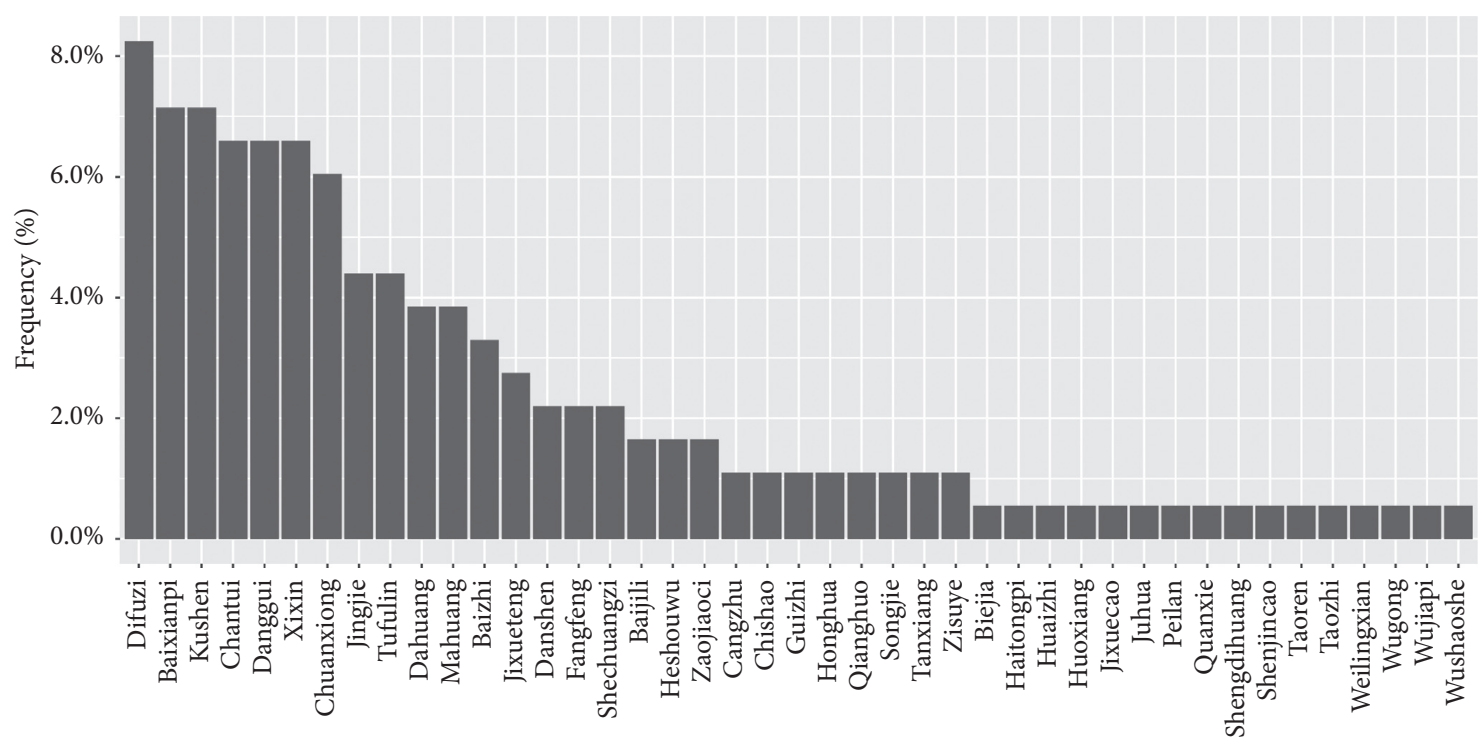

Herb

FIGURE 1: Frequency distribution of herbs used in the 17 RCTs included in the meta-analysis on CHBT treatment for UP. 
TABle 2: The Chinese herbs mentioned in the article.

\begin{tabular}{|c|c|c|c|}
\hline Chinese name & English name & Latin name & Frequency of usage \\
\hline \multicolumn{4}{|c|}{ The 43 Chinese herbs used in 17 prescriptions of CHBT } \\
\hline Difuzi & Fructus kochiae & Bassia scoparia (L.) A.J.Scott & 15 \\
\hline Baixianpi & Densefruit pittany root-bark & Dictamnus albus L. & 13 \\
\hline Kushen & Lightyellow sophora & Sophora flavescens aiton & 13 \\
\hline Danggui & Chinese angelica & Angelica sinensis (oliv.) diels & 12 \\
\hline Xixin & Manchurian wildginger & Asarum sieboldii miq. & 12 \\
\hline Chantui & Cicada slough & Cryptotympana atrata fabr. & 12 \\
\hline Chuanxiong & Szechuan lovage rhizome & Ligusticum striatum DC. & 11 \\
\hline Jingjie & Fineleaf schizonepeta herb & Nepeta tenuifolia benth & 8 \\
\hline Tufulin & Rhizoma smilacis glabrae & Smilax glabra roxb & 8 \\
\hline Dahuang & Rhubarb root and rhizome & Rheum officinale baill. & 7 \\
\hline Mahuang & Ephedra equisetina & Ephedra sinica stapf & 7 \\
\hline Baizhi & Dahurian angelica & Bletilla striata (thunb.) Rchb.f. & 6 \\
\hline Jixueteng & Suberect spatholobus stem & Millettia dielsiana harms & 5 \\
\hline Danshen & Salvia miltiorrhiza $f$ alba & Salvia miltiorrhiza bunge & 4 \\
\hline Shechuangzi & Common cnidium & Cnidium monnieri (L.) cusson & 4 \\
\hline Fangfeng & Divaricate saposhnikovia & Saposhnikovia divaricata (turcz.) schischk. & 4 \\
\hline Heshouwu & Tuber fleeceflower & Reynoutria multiflora (Thunb.) moldenke & 3 \\
\hline Zaojiaoci & Spine of Chinese honeylocust & Gleditsia sinensis lam. & 3 \\
\hline Baijili & Tribulus fruit & Tribulus terrestris $L$. & 3 \\
\hline Cangzhu & Atractylodes japonica & Atractylodes lancea (thunb.) DC. & 2 \\
\hline Guizhi & Cassiabarktree twig & Cinnamomum cassia (L.) J.Presl & 2 \\
\hline Honghua & Safflower & Carthamus tinctorius L. & 2 \\
\hline Zisuye & Perilla frutescens var acuta & Perilla frutescens (L.) britton & 2 \\
\hline Songjie & Knotty pine wood & Pinus tabuliformis carrière & 2 \\
\hline Chishao & Red peony root & Paeonia lactiflora pall. & 2 \\
\hline Qianghuo & Notopterygium forbesii & Notopterygium incisum K.C.Ting ex H.T.Chang & 2 \\
\hline Tanxiang & Sandalwood & Santalum album L. & 2 \\
\hline Haitongpi & Oriental Variegated coralbean bark & Erythrina variegata $\mathrm{L}$. & 1 \\
\hline Huaizhi & Twig of Japanese pagodatree & Styphnolobium japonicum (L.) schott & 1 \\
\hline Wugong & Centipede & Scolopendra Subspinipes Mutilans & 1 \\
\hline Wushaoshe & Chinese rat snake & Zaocys dhumnades cantor & 1 \\
\hline Biejia & Turtle shell & Trionyx sinensis wiegmann & 1 \\
\hline Huoxiang & Wrinkled gianthyssop & Pogostemon cablin (blanco) benth. & 1 \\
\hline Peilan & Eupatorium formosanum & Eupatorium fortunei turcz. & 1 \\
\hline Taoren & Peach kernel & Prunus persica (L.) batsch & 1 \\
\hline Juhua & Florists chrysanthemum flower & Chrysanthemum morifolium ramat. & 1 \\
\hline Wujiapi & Acanthopanax senticosus & Eleutherococcus nodiflorus (dunn) S.Y.Hu & 1 \\
\hline Shengdihuang & Fresh rehmannia root & Rehmannia glutinosa (gaertn.) DC. & 1 \\
\hline Weilingxian & Chinese clematis & Clematis chinensis osbeck & 1 \\
\hline Quanxie & Scorpion & Buthus martensii karsch. & 1 \\
\hline Jixuecao & Asiatic pennywort & Centella asiatica (L.) urb. & 1 \\
\hline Taozhi & Prunus davidiana & Prunus persica (L.) batsch & 1 \\
\hline Shenjincao & Common clubmoss herb & Lycopodium clavatum L. & 1 \\
\hline \multicolumn{4}{|c|}{ The other Chinese herbs mentioned in the article } \\
\hline Sangbaipi & Folium mori albae & Morus alba L. & - \\
\hline Zicao & Radix arnebiae & Lithospermum erythrorhizon siebold and zucc. & - \\
\hline Huangqi & Radix astragali & Astragalus henryi oliv. & - \\
\hline Yizhiren & Sharpleaf galangal & Alpinia oxyphylla miq. & - \\
\hline Wuweizi & Schisandra sphenanthera & Schisandra chinensis (turcz.) baill. & - \\
\hline
\end{tabular}

$\mathrm{CHBT}=$ Chinese herbal bath therapy.

elimination of bioactive ingredients, compared to either Schisandra chinensis or Alpinia oxyphylla alone, in a rat model of Alzheimer's disease [45]. Luo et al. have reported that Sprague-Dawley rats orally administered the Ephedra sinica Stapf-Cinnamomum cassia (L.) J. Presl herb pair could improve the pharmacological effects of Ephedra sinica Stapf and reduce its toxicity (apparent when used alone) by reducing tissue accumulation of ephedrine alkaloids [46]. Hence, herb pairs appear to show greater pharmacological efficacy than a single herb.

Research using modern pharmacological methods has demonstrated that the core herb combinations listed here could improve UP through their anti-inflammatory, antioxidant, and antibacterial properties and by accelerating 


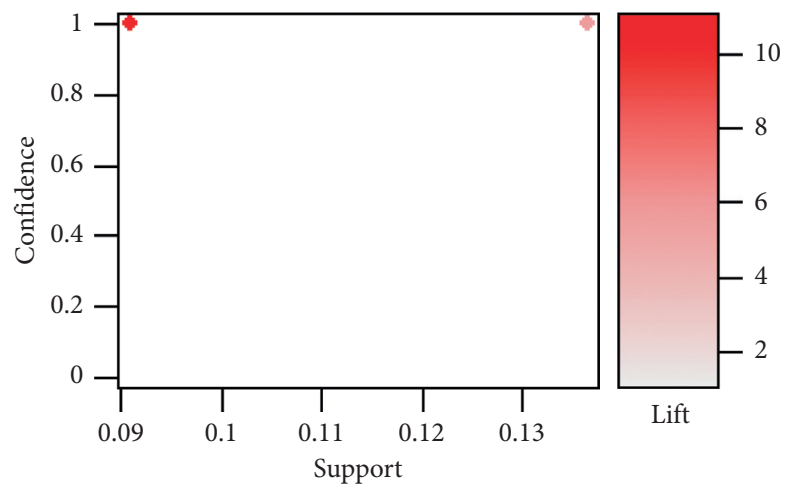

FIGURE 2: Scatter plot for the 19 association rules obtained in the 17 RCTs included in the meta-analysis on CHBT treatment for UP.

TABLE 3: Apriori algorithm-based association rules for herbs used in CHBT.

\begin{tabular}{|c|c|c|c|c|c|}
\hline No & Association rules & Support & Confidence & Expected confidence & Lift \\
\hline 1 & $\{$ Jixueteng $\} \geq\{$ danggui $\}$ & 0.13636364 & 1.000000 & 0.181818182 & 5.500000 \\
\hline 2 & $\{$ Jixueteng $\} \geq\{$ chantui $\}$ & 0.13636364 & 1.000000 & 0.318181833 & 3.142857 \\
\hline 3 & $\{$ Baixianpi $\} \geq\{$ difuzi $\}$ & 0.13636364 & 1.000000 & 0.181818182 & 5.500000 \\
\hline 4 & $\{$ Chuanxiong $\} \geq\{$ dahuang $\}$ & 0.09090909 & 1.000000 & 0.090909091 & 11.000000 \\
\hline 5 & $\{$ Dahuang $\} \geq\{$ chuanxiong $\}$ & 0.09090909 & 1.000000 & 0.090909091 & 11.000000 \\
\hline 6 & $\{$ Chuanxiong $\} \geq\{$ baizhi $\}$ & 0.09090909 & 1.000000 & 0.136363643 & 7.333333 \\
\hline 7 & $\{$ Dahuang $\} \geq\{$ baizhi $\}$ & 0.09090909 & 1.000000 & 0.136363643 & 7.333333 \\
\hline 8 & $\{$ Baijili $\} \geq\{$ baizhi $\}$ & 0.09090909 & 1.000000 & 0.136363643 & 7.333333 \\
\hline 9 & $\{$ Baijili $\} \geq\{$ baixianpi $\}$ & 0.09090909 & 1.000000 & 0.136363643 & 7.333333 \\
\hline 10 & $\{$ Baijili $\} \geq\{$ danshen $\}$ & 0.09090909 & 1.000000 & 0.136363643 & 7.333333 \\
\hline 11 & $\{$ Baijili $\} \geq\{$ difuzi $\}$ & 0.09090909 & 1.000000 & 0.181818182 & 5.500000 \\
\hline 12 & $\{$ Guizhi $\} \geq\{$ chantui $\}$ & 0.09090909 & 1.000000 & 0.318181833 & 3.142857 \\
\hline 13 & $\{$ Songjie $\} \geq\{$ kushen $\}$ & 0.09090909 & 1.000000 & 0.181818182 & 5.500000 \\
\hline 14 & $\{$ Chishao $\} \geq\{$ difuzi $\}$ & 0.09090909 & 1.000000 & 0.181818182 & 5.500000 \\
\hline 15 & $\{$ Chishao $\} \geq\{$ kushen $\}$ & 0.09090909 & 1.000000 & 0.181818182 & 5.500000 \\
\hline 16 & $\{$ Heshouwu $\} \geq\{$ fangfeng $\}$ & 0.09090909 & 1.000000 & 0.136363643 & 7.333333 \\
\hline 17 & $\{$ Heshouwu $\} \geq\{$ jingjie $\}$ & 0.09090909 & 1.000000 & 0.181818182 & 5.500000 \\
\hline 18 & $\{$ Heshouwu $\} \geq\{$ kushen $\}$ & 0.09090909 & 1.000000 & 0.181818182 & 5.500000 \\
\hline 19 & $\{$ Heshouwu $\} \geq\{$ chantui $\}$ & 0.09090909 & 1.000000 & 0.318181833 & 3.142857 \\
\hline
\end{tabular}

CHBT $=$ Chinese herbal bath therapy.

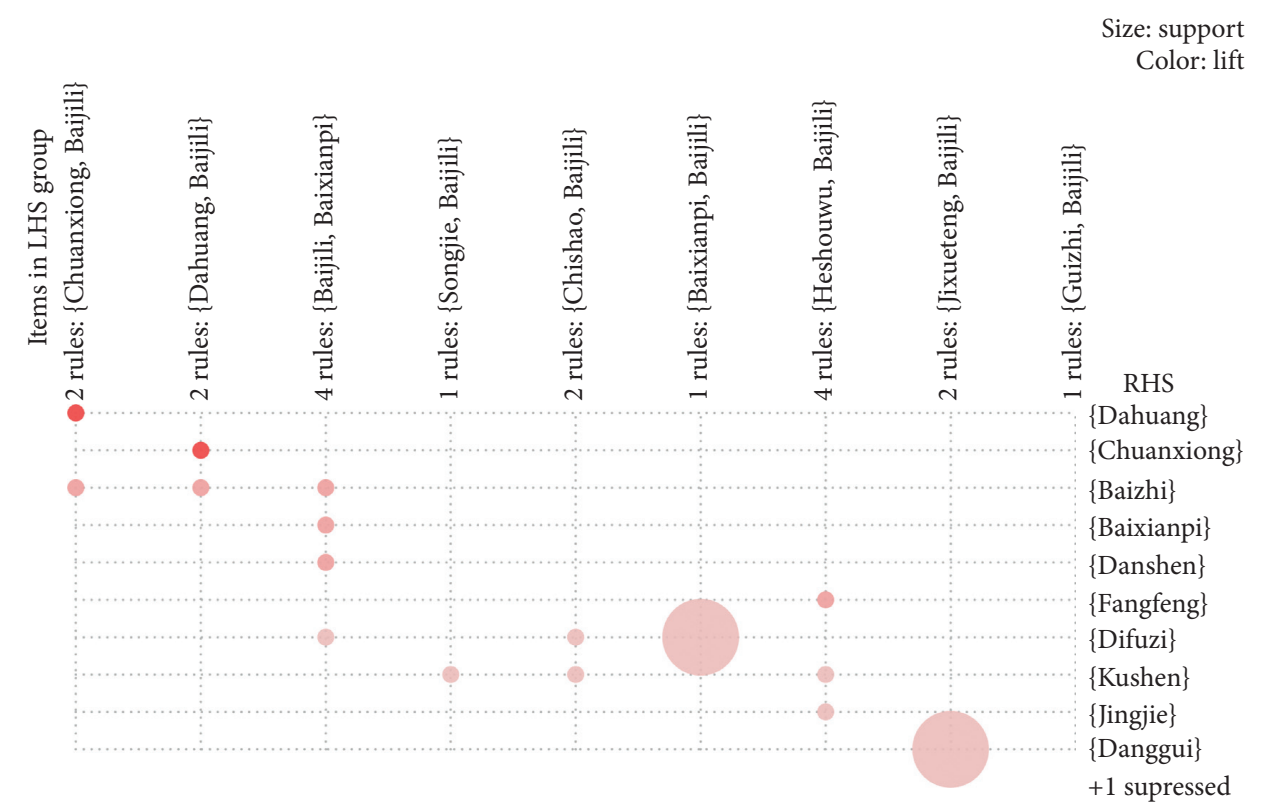

FIgURE 3: Grouping matrix for the 19 association rules obtained in the 17 RCTs included in the meta-analysis on CHBT treatment for UP. 
TABLE 4: Potential efficacy of the core Chinese medicinal herbs of CHBT used for the treatment of uremic pruritus.

\begin{tabular}{lcccc}
\hline Chinese name & English/Latin name & Function & Active ingredients & Efficacy \\
\hline Baijili [48, 50] & $\begin{array}{c}\text { Tribulus fruit/Tribulus } \\
\text { terrestris } L .\end{array}$ & Pruritus relief & $\begin{array}{c}\text { Saponins, flavonoids, } \\
\text { glycosides }\end{array}$ & $\begin{array}{c}\text { Antihelmintic, antibacterial, and } \\
\text { antiinflammatory activity }\end{array}$ \\
\hline $\begin{array}{l}\text { Chuanxiong } \\
{[47,51]}\end{array}$ & $\begin{array}{c}\text { Szechuan lovage rhizome/ } \\
\text { Ligusticum striatum DC. }\end{array}$ & $\begin{array}{c}\text { Activating blood } \\
\text { circulation }\end{array}$ & $\begin{array}{c}\text { Ferulic acid, senkyunolide, } \\
\text { butylidenephthalide }\end{array}$ & $\begin{array}{c}\text { Antithrombotic, antihypertensive, } \\
\text { and vasodilator activity }\end{array}$ \\
\hline Dahuang [49] & $\begin{array}{c}\text { Rhubarb root and } \\
\text { rhizome/Rheum officinale } \\
\text { baill. }\end{array}$ & $\begin{array}{c}\text { Promoting } \\
\text { detoxification }\end{array}$ & $\begin{array}{c}\text { Anthraquinones, Stilbenes } \\
\text { Antibacterial, anti-inflammatory, and } \\
\text { free-radical scavenging activity }\end{array}$
\end{tabular}

CHBT $=$ Chinese herbal bath therapy.

blood circulation to promote metabolism and eliminating uremia toxins [8, 47-51] (Table 4).

\section{Conclusions}

Chinese medicinal formulas that use combinations of many herbs represent potential therapeutic options for some conditions; however, data on their reliability and mechanism of action are lacking. Our study shows the combinations of herbs used in Chinese medicine formulas, such as Chuanxiong, Baijili, and Dahuang, and Dahuang, Baijili, and Chuanxiong, may be potentially useful in the treatment of UP. Further experiments are needed to confirm these observations and to explore their mechanism(s) of action.

5.1. Limitations. Despite our results on potential core herb combinations for the treatment of UP, this study has a few limitations. First, the overall risk of bias was high in most RCTs included in the meta-analysis and none of the studies mentioned double-blinding. Thus, high RoB should be considered when interpreting these results. Next, most of the included RCTs did not have careful follow-up protocols, and the treatment duration only lasts two weeks in some RCTs. Hence, future studies should focus on establishing the longterm efficacy and safety of these core prescriptions for UP. Finally, as the mechanisms of action of these herb combinations are unclear, further basic and clinical studies are also needed.

\section{Data Availability}

The data used to support the findings of this study are included within the article.

\section{Conflicts of Interest}

The authors declare that there are no conflicts of interest regarding the publication of this article.

\section{Acknowledgments}

The authors thank Taipei Tzu Chi Hospital and the participants for their contribution to the study. This study was supported by Taipei Tzu Chi Hospital and Buddhist Tzu Chi Medical Foundation, New Taipei City, Taiwan (TCRDTPE-109-12).

\section{Supplementary Materials}

Supplementary Figure 1: risk of bias assessment of the retrieved RCTs. (Supplementary Materials)

\section{References}

[1] X. Hu, Y. Sang, M. Yang, X. Chen, and W. Tang, "Prevalence of chronic kidney disease-associated pruritus among adult dialysis patients: a meta-analysis of cross-sectional studies," Medicine (Baltimore), vol. 97, no. 21, Article ID e10633, 2018.

[2] N. Sukul, E. Speyer, C. Tu et al., "Pruritus and patient reported outcomes in non-dialysis CKD," Clinical Journal of the American Society of Nephrology, vol. 14, no. 5, pp. 673-681, 2019.

[3] M. Forouzandeh, S. Stratman, and G. Yosipovitch, "The treatment of kyrle's disease: a systematic review," Journal of the European Academy of Dermatology and Venereology, vol. 34, no. 7, pp. 1457-1463, 2020.

[4] K. Grochulska, R. Ofenloch, T. Mettang, and E. Weisshaar, "Mortality of haemodialysis patients with and without chronic itch: a follow-up study of the German epidemiological hemodialysis itch study (GEHIS)," Acta Dermato Venereologica, vol. 99, no. 4, pp. 423-428, 2019.

[5] M. K. Fallahzadeh, J. Roozbeh, B. Geramizadeh, and M. R. Namazi, "Interleukin-2 serum levels are elevated in patients with uremic pruritus: a novel finding with practical implications," Nephrology Dialysis Transplantation, vol. 26, no. 10, pp. 3338-3344, 2011.

[6] M. Kimmel, D. M. Alscher, R. Dunst et al., "The role of microinflammation in the pathogenesis of uraemic pruritus in haemodialysis patients," Nephrology Dialysis Transplantation, vol. 21, no. 3, pp. 749-755, 2006.

[7] T. Ye, W. Tu, and G. Xu, "Hot bath for the treatment of chronic renal failure," Renal Failure, vol. 36, no. 1, pp. 126-130, 2014.

[8] W. Xue, Y. Zhao, M. Yuan, and Z. Zhao, "Chinese herbal bath therapy for the treatment of uremic pruritus: meta-analysis of randomized controlled trials," BMC Complementary and Alternative Medicine, vol. 19, no. 1, p. 103, 2019.

[9] X. Chen, L.-J. Li, and D. Wu, "A clinical study on uremic clearance granule combined with high-flux hemodialysis in treating uremia pruritus," China Pharmaceuticals, vol. 4, pp. 63-65, 2020.

[10] X. Zhang, M. Zhang, Z. Yang et al., "A study on the antiinflammation and analgesic effects of nature of anti-rheumatism traditional Chinese medicine," Chinese Archives of Traditional Chinese Medicine, vol. 26, pp. 2386-2396, 2008.

[11] L. Zou, F. Fang, G. Tu et al., "Effect of achyranthes bidentata polysaccharides on hemorrheology of rabbit's knee osteoarthritis," Henan Journal of Traditional Chinese Medicine, vol. 33, pp. 2083-2085, 2013. 
[12] L. L. Liu and J. Duan, "A study about the elimination of uremia toxins by traditional Chinese medicine bath therapy from the theory of "opening ghost door", Journal of New Chinese Medicine, vol. 45, no. 7, pp. 189-190, 2013.

[13] Y. Yanna, L. Wang, and X. Wu, "Curative effect observation of applying dispelling wind and dampness itching medicated bath therapy for uremic prutitus," Journal of New Chinese Medicine, no. 12, p. 22, 2017.

[14] L. Xu, X. Li, and W. Wang, Chinese Materia Medica: Combinations and Applications, Donica, UK, 2002.

[15] B. Chen, H. Zhan, M. Chung et al., "Chinese herbal Bath therapy for the treatment of knee osteoarthritis: meta-analysis of randomized controlled trials," Evidence-Based Complementary and Alternative Medicine, vol. 2015, p. 12, Article ID 949172, 2015.

[16] P. Xia, K. Gao, J. Xie et al., "Data mining-based analysis of Chinese medicinal herb formulae in chronic kidney disease treatment," Evidence-Based Complementary and Alternative Medicine, vol. 2020, p. 14, Article ID 9719872, 2020.

[17] X. You, Y. Xu, J. Huang et al., "A data mining-based analysis of medication rules in treating bone marrow suppression by kidney-tonifying method," Evidence-Based Complementary and Alternative Medicine, vol. 2019, p. 9, Article ID 1907848, 2019.

[18] Y. He, X. Zheng, C. Sit et al., "Using association rules mining to explore pattern of Chinese medicinal formulae (prescription) in treating and preventing breast cancer recurrence and metastasis," Journal of Translational Medicine, vol. 10, no. Suppl 1, p. S12, 2012.

[19] R. J. Bayardo and R. Agrawal, Eds., in Proceedings of the FifthACM SIGKDD International Conference on Knowledge Discovery and Data Mining, Association for Computing Machinery, pp. 145-154, San Diego, CA, USA, 1999.

[20] J. P. Higgins, J. A. Sterne, J. Savovic et al., "A revised tool for assessing risk of bias in randomized trials," Cochrane Database of Systematic Reviews, vol. 10, no. Suppl 1, pp. 29-31, 2016.

[21] J. Verzani, Getting Started with RStudio:, O’Reilly Media, Inc., Sebastopol, CA, USA, 2011.

[22] M. J. Zaki, S. Parthasarathy, L. Wei, and M. Ogihara, "Evaluation of sampling for data mining of association rules," in Proceedings of the Seventh International Workshop on Research Issues in Data Engineering High Performance Database Management for Large-Scale Applications, Birmingham, UK, April 1997.

[23] W. Du, "Clinical observation on the treatment of uremia combined with pruritus of skin by Chinese herbal bath," Chinese Community Doctors, vol. 11, no. 22, p. 122, 2009.

[24] M. J. Du, Y. W. Wen, and L. H. Sun, "Chinese herbal bath for treating 15 patients with uremic pruritus," Clinical Journal of Traditional Chinese Medicine, no. 2, p. 126, 2004.

[25] J. M. Gao and J. Y. Ye, "Clinical observation of 21 patients of uremic pruritus treated by Chinese herbal bath," Clinical Focus, vol. 27, no. 3, pp. 257-258, 2012.

[26] X. L. Guo, J. C. Tan, and G. Wang, "Clinical observation of skin pruritus in hemodialysis patients treated by traditional Chinese herbal bath," China Modern Medicine, vol. 16, no. 7, pp. 84-85, 2009.

[27] Y. L. Jia, X. M. Li, and Y. L. Fei, "Effect of Shengshizhiyang Tang on uremia itchy skin," Journal of Hainan Medical University, vol. 18, no. 5, pp. 646-647, 2012.

[28] Y. L. Jia, X. M. Li, J. H. Du, and Y. Chang, "Observation on curative effect of treating uremic pruritus with
Shengshizhiyang Tang," Journal of Hebei Medical University, vol. 33, no. 12, pp. 1434-1436, 2012.

[29] S. Y. Lan, "Analysis on the effect of Chinese herb medicated bath in the treatment of cutaneous pruritus of maintenance hemodialysis," Guangming Traditional Chinese Medicine, vol. 32, no. 19, pp. 2791-2793, 2017.

[30] Y. F. Lin, "Clinical efficacy and safety analysis of Shengshizhiyang Tang washing for treating patients with uremic pruritus," Chinese Journal of Traditional Medical Science and Technology, no. z2, p. 32, 2014.

[31] H. Shen, "Clinical observation on the effect of Chinese herbal bath on relieving pruritus in hemodialysis patients," World Health Digest, no. 25, pp. 285-286, 2014.

[32] L. Y. Wang, J. S. Zhao, M. Luo, F. L. Wang, and M. Yang, "Clinical observation of Chinese herbal bath for treating 27 patients with uremic pruritus," Chinese Medicine Modern Distance Education of China, no. 20, p. 35, 2013.

[33] Y. W. Wen, "Chinese herbal bath for treating 21 hemodialysis patients with uremic pruritus," Journal of Emergency in Traditional Chinese Medicine, no. 5, p. 623, 2007.

[34] L. Yao, "TCM formula granule washing for treating for 40 patients with uremic pruritus," China Pharmaceuticals, no. B11, pp. 65-66, 2015.

[35] Y. N. Yu, L. L. Wang, and X. B. Wu, "Curative effect observation of applying dispelling wind and dampness itching medicated bath therapy for uremic pruritus," Journal of New Chinese Medicine, no. 12, pp. 64-66, 2017.

[36] C. L. Zhang, G. Z. Yu, X. Q. Gui, C. E. Huang, and $\mathrm{X}$. W. Zhang, "Experience of nursing uremic pruritus with Chinese medicine formula granule," Chinese Medicine Modern Distance Education of China, vol. 12, no. 20, pp. 128-129, 2014.

[37] Y. L. Zhang, H. F. Zhu, S. Z. Wei, and L. Li, "Clinical observation of Chinese herbal bath for treating 78 patients with uremic pruritus," World Health Digest, no. 41, pp. 382-383, 2012.

[38] H. Zhao, Open Cou Discharge Turbidity Dipping Treatments for Uremia Itchy Skin Clinical Research, Master Nanjing University of Chinese Medicine, Nanjing, China, 2011.

[39] J. Zheng, "Observation on the curative effect of Yangxuezhiyang Mixture on uremia patients," Contmporary Medicine, no. 24 , pp. $160-161,2016$.

[40] C.-M. Kao and J.-H. Li, "The observation on the efficacy of Niaoduqing retention enema combined with low-frequency hemoperfusion and hemodialysis on uremia pruritus," China Health Care and Nutrition, vol. 22, no. 7, pp. 1846-1847, 2012.

[41] P.-G. Wang, "Xiao Feng san addition and subtraction treatment 30 cases of uremic pruritus," Journal of Practical Traditional Chinese Internal Medicine, vol. 9, pp. 70-71, 2010.

[42] Y. Bai, H. Jia, and L. Zhang, "Analysis of clinical effect of lifu paste in treating patients of long-term dialysis complicated with cutaneous pruritus," Zhongguo Zhong Xi Yi Jie He Za Zhi Zhongguo Zhongxiyi Jiehe Zazhi= Chinese Journal of Integrated Traditional and Western Medicine, vol. 22, no. 4, pp. 301-302, 2002.

[43] M. X. Sheng, J. Z. Li, and H. Y. Wang, "Therapeutic effect of Astragalus and Angelica on renal injury induced by ischemia/ reperfusion in rats," Zhongguo Zhong Xi Yi Jie He Za Zhi Zhongguo Zhongxiyi Jiehe Zazhi = Chinese Journal of Integrated Traditional and Western Medicine, vol. 21, no. 1, pp. 43-46, 2003.

[44] J. Song, L. Meng, S. Li, L. Qu, and X. Li, “A combination of Chinese herbs, Astragalus membranaceus var. mongholicus and Angelica sinensis, improved renal microvascular 
insufficiency in 5/6 nephrectomized rats," Vascular Pharmacology, vol. 50, no. 5-6, pp. 185-193, 2009.

[45] Y. Qi, X. Cheng, H. Jing et al., "Comparative pharmacokinetic study of the components in Alpinia oxyphylla Miq.-Schisandra chinensis (Turcz.) Baill. herb pair and its single herb between normal and Alzheimer's disease rats by UPLC-MS/ MS," Journal of Pharmaceutical and Biomedical Analysis, vol. 177, Article ID 112874, 2020.

[46] P. Wei, H.-l. Huo, Q.-h. Ma et al., "Pharmacokinetic comparisons of five ephedrine alkaloids following oral administration of four different Mahuang-Guizhi herb-pair aqueous extracts ratios in rats," Journal of Ethnopharmacology, vol. 155, no. 1, pp. 642-648, 2014.

[47] X. Ran, L. Ma, C. Peng, H. Zhang, and L.-P. Qin, "Ligusticum chuanxiongHort: a review of chemistry and pharmacology," Pharmaceutical Biology, vol. 49, no. 11, pp. 1180-1189, 2011.

[48] S. Chhatre, T. Nesari, D. Kanchan, G. Somani, and S. Sathaye, "Phytopharmacological overview of Tribulus terrestris," Pharmacognosy Reviews, vol. 8, no. 15, pp. 45-51, 2014.

[49] Y. J. Cao, Z. J. Pu, Y. P. Tang et al., "Advances in bio-active constituents, pharmacology and clinical applications of rhubarb," Chinese Medicine, vol. 12, p. 36, 2017.

[50] W. Zhu, Y. Du, H. Meng, Y. Dong, and L. Li, "A review of traditional pharmacological uses, phytochemistry, and pharmacological activities of Tribulus terrestris," Chemistry Central Journal, vol. 11, no. 1, p. 60, 2017.

[51] Z. Chen, C. Zhang, F. Gao et al., "A systematic review on the rhizome of Ligusticum chuanxiong Hort. (Chuanxiong)," Food and Chemical Toxicology, vol. 119, pp. 309-325, 2018. 